\title{
Metodologia de Discretização do Método dos Elementos de Contorno em Problemas Tridimensionais
}

\author{
Barbosa, J. P. ${ }^{1,2^{*}}$; Loeffler, C. F. \\ 1 Programa de Pós-Graduação em Engenharia Mecânica, Universidade Federal do Espírito Santo, Vitória, ES, Brasil. \\ 2 Instituto Federal do Espírito Santo - IFES Campus São Mateus, ES, Brasil, ES, Brasil. \\ *e-mail: jpbarbosa@ifes.edu.br
}

\begin{abstract}
Resumo
Neste trabalho apresenta-se detalhadamente a metodologia de discretização do Método de Elementos de Contorno tridimensional (MEC) na abordagem de problemas homogêneos governados pela Equação de Laplace. Não obstante seus princípios matemáticos serem bem conhecidos e o MEC estar consolidado no meio científico devido as bem sucedidas aplicações em diversos campos da física e da engenharia, as diversas particularidades que cercam seu modelo numérico em problemas tridimensionais não se encontram acessíveis. Assim, especificidades referentes aos procedimentos de integração, que envolvem transformações de coordenadas e 0 trato com funções singulares, são aqui apresentadas detalhadamente, pois não se encontram na literatura. A discretização é feita através de elementos isoparamétricos triangulares planos, de variação linear, com nós duplos nos cantos. As integrais sobre os elementos são calculadas de uma forma mista: analítica e numérica. Resolve-se um exemplo simples, apresentando-se um gráfico de convergência de erros, mostrando o decréscimo destes com o refinamento da malha, ratificando assim a consistência da implementação computacional gerada a partir do modelo discreto proposto.
\end{abstract}

\begin{abstract}
This work presents the methodology of discretization of the Three-Dimensional Boundary Element Method (BEM) to solve homogeneous problems governed by the Laplace Equation. The boundary integrals are discretized using linear triangular isoparametric elements with linear variation. The integrals on the elements are calculated in a mixed form: analytical and numerical. The field function and its derivative in a normal direction are interpolated using also a linear function on the surface of boundary elements. Several particularities, especially concerning analytical and numerical integrations, are presented here in detail, since the specialized literature does not approach suitably three dimensional numerical modeling. An example is solved by presenting a graph of convergence of errors, showing the decrease of these with the refinement of the mesh, thus ratifying the consistency of the computational implementation.
\end{abstract}

Keywords (Palavras chaves): Elementos de Contorno, Elementos Triangulares, Problemas Tridimensional, Equação de Laplace.

\section{Introdução}

Vários problemas físicos, como os que representam a transferência de calor, a condução elétrica em corrente contínua ou a percolação de fluidos em meios porosos são regidos pela Equação de Laplace. Esta equação tem grande importância na Física Matemática, não só por descrever uma série de fenômenos estacionários, mas também por servir de base para solução de outras equações mais complicadas, como as Equações de Helmholtz, da Difusão-Advecção e da Onda Acústica.
Atualmente, devido à maior abrangência, as soluções da Equação de Laplace por meio de métodos analíticos têm dado lugar às soluções aproximadas, obtidas por métodos numéricos, que oferecem a possibilidade de solucionar problemas complexos em tempo reduzido, pois seus cálculos são feitos computacionalmente. Estão entre os métodos numéricos mais utilizados: o Método dos Elementos de Contorno (MEC) [1], o Método dos Elementos Finitos (MEF) [2] e o Método das Diferenças Finitas (MDF) [3].

Diferentemente dos demais, o MEC tem como principal característica a transformação da equação diferencial 
que governa o problema numa equação integral de contorno [4]. Assim, o MEC efetua a redução do problema em uma dimensão, isto é, somente pontos situados no contorno figuram no processo de modelagem numérica. Num caso tridimensional, a aproximação do meio contínuo se faz por elementos de superfície triangulares, geometricamente definidos por pontos nodais em seus vértices.

Neste trabalho, cujo principal objetivo consiste no detalhamento do modelo discreto e na operacionalização da sua solução computacional, a integração sobre os elementos é feita tanto analiticamente quanto numericamente, dependendo da localização dos pontos de geração das equações de contorno, pontos estes que são argumentos de uma função auxiliar, denominada solução fundamental [5]. Esta função é de grande importância na definição das características e potencialidades do MEC. Se esses pontos fonte pertencerem ao elemento no qual se calculam as integrais, faz-se o cálculo analítico; quando estes estão localizados noutros elementos, as integrais são calculadas numericamente, utilizando-se as fórmulas da Quadratura Gaussiana [6,7].

\section{Equação Integral de Contorno}

Considera-se um domínio tridimensional $V(X)$ que pode representar um campo térmico ou mecânico no estado estacionário com propriedades homogêneas e isotrópicas. Também se admite não haver fontes, sorvedouros ou ações de domínio externo que contribuam diretamente para o campo [8].

Sendo a variável básica $u(X)$ o potencial escalar em um dado ponto $X=X\left(x_{1}, x_{2}, x_{3}\right)$, o domínio $V(X)$ é limitado por uma superfície de contorno $S(X)$, com condições de contorno essenciais $u=\bar{u}$ no contorno $S_{l}$ e naturais $\nabla u=\bar{q}$ no contorno $S_{2}$, onde $\bar{u}$ e $\bar{q}$ são valores conhecidos de $u$ e $\nabla u$, a equação diferencial associada a este problema é a Equação de Laplace:

$\nabla^{2} u=0$

Através dos princípios da Teoria das Equações Integrais chega-se a seguinte equação integral:

$c(P) u(P)+\int_{S} u(X) q^{*}(\xi ; X) d S=\int_{S} q(X) u^{*}(\xi ; X) d S$

$\mathrm{Na}$ equação anterior, a função auxiliar $u^{*}(P ; X)$ é a solução fundamental e sua derivada normal é expressa por $q^{*}(P ; X)$. Tais funções dependem da distância euclidiana entre dois pontos, um deles o já mencionado ponto de geração das equações ou ponto fonte $P$. $O$ coeficiente $c(P)$ depende da suavidade do contorno [1].

\subsection{Interpolação do Campo e da Geometria}

A formulação do MEC não requer integrações tridimensionais, mas envolve funções singulares. Uma estratégia para o cálculo das integrais ao longo da superfície $S$ é a divisão da superfície conforme figura 1 em uma soma de superfícies menores $S_{1}, S_{2}, S_{3}, \ldots, S_{n}$, como mostra a equação (3), onde $n$ é o número de superfícies em que o contorno foi dividido.

$S=\sum_{i=1}^{n} S_{i}$

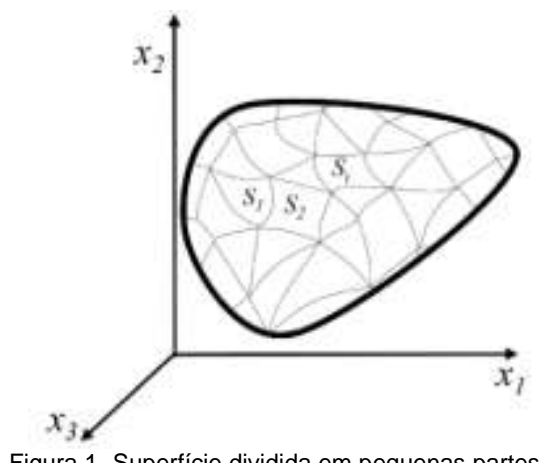

Figura 1- Superfície dividida em pequenas partes.

Assim, a equação (2), pode ser escrita como:

$c(P) u(P)+\sum_{j=1}^{n} \int_{S_{j}} u q^{*} d S-\sum_{j=1}^{n} \int_{S_{j}} q u^{*} d S$

Omitindo-se o argumento das funções, as variáveis $u$ e $q$ sobre cada elemento $j$ são definidas em termos de valores nodais:

$u=\left[\begin{array}{llll}\phi_{1} & \phi_{2} & \Lambda & \phi_{n}\end{array}\right]\left\{\begin{array}{c}u^{1} \\ u^{2} \\ \mathrm{M} \\ u^{n}\end{array}\right\} \quad q=\left[\begin{array}{llll}\phi_{1} & \phi_{2} & \Lambda & \phi_{n}\end{array}\right]\left\{\begin{array}{c}q^{1} \\ q^{2} \\ \mathrm{M} \\ q^{n}\end{array}\right\}$

Nas equações anteriores $\phi_{i}$ são as funções de forma ou funções de interpolação, que são dadas em termos de coordenadas adimensionais $\eta(x)$ sobre cada elemento. É usual escrever-se tanto a variação do campo quanto a geometria do corpo através dessas funções $[2,4]$. Logo, as coordenadas $\left(x_{1}, x_{2}, x_{3}\right)$ de um ponto qualquer do elemento são escritas em função das coordenadas dos $N$ nós do elemento.

Embora as superfícies elementares possam ter uma forma qualquer, aqui são formadas por triângulos, o que introduz uma aproximação na superfície original, especialmente se esta não for plana. No entanto, os triângulos planos são mais fáceis de gerar e possuem Jacobiano constante ao longo do elemento.

\section{Metodologia de Discretização}





a) Espaço Real - $\left(x_{1}, x_{2}, x_{3}\right)$; b) Espaço Paramétrico - $\left(\eta_{1}, \eta_{2}\right)$. Figura 2 - Elemento triangular.

Uma vez que a integração de Gauss será realizada no intervalo $[0,1]$, por simplicidade é coerente fazer o mapeamento do elemento $S_{i}$ também no intervalo [0,1]. Deste modo, na figura 2 observa-se o elemento real e o elemento de integração, onde se definem as seguintes relações:

$$
\phi_{1}=\eta_{1} ; \quad \phi_{2}=\eta_{2} ; \quad \phi_{3}=1-\eta_{1}-\eta_{2}
$$

Os pontos nodais de integração são:

$$
X_{i}=\sum_{n=1}^{3} \phi^{n} X_{i}^{n}
$$

Na equação (7) $X_{i}^{n}$ é a coordenada cartesiana do nó $n$. Em elementos triangulares planos as funções $u$ e $q$ variam linearmente sobre tais elementos.

$$
[\phi]=\left[\begin{array}{lll}
\eta_{1} & \eta_{2} & \eta_{3}
\end{array}\right]
$$

Desta forma, pode-se escrever:

$$
\begin{aligned}
& {[\hat{H}]_{i j}=\int_{S_{j}}[\phi] q^{*} d S=\int_{S_{j}}\left[\begin{array}{lll}
\eta_{1} & \eta_{2} & \eta_{3}
\end{array}\right] q^{*} d S} \\
& {[G]_{i j}=\int_{S_{j}}[\phi] u^{*} d S=\int_{S_{j}}\left[\begin{array}{lll}
\eta_{1} & \eta_{2} & \eta_{3}
\end{array}\right] u^{*} d S}
\end{aligned}
$$

É necessário relacionar $d S$, representado no sistema de coordenadas globais $X$, com tal sistema de coordenadas, através do Jacobiano " $J$ " da transformação de coordenadas, dado por:

$$
d S=|J| d \eta_{1} d \eta_{2}
$$

Demonstra-se [9] que Jacobiano da transformação é dado por:

$|J|=\left(\frac{\partial x_{1}}{\partial \eta_{1}}, \frac{\partial x_{2}}{\partial \eta_{1}}, \frac{\partial x_{3}}{\partial \eta_{1}}\right) \times\left(\frac{\partial x_{1}}{\partial \eta_{2}}, \frac{\partial x_{2}}{\partial \eta_{2}}, \frac{\partial x_{3}}{\partial \eta_{2}}\right) \mid$

Observa-se que as diferenciais representam as coordenadas dos vetores formados por dois lados adjacentes do triângulo, de modo o Jacobiano é igual ao módulo do produto vetorial desses dois vetores, numericamente igual ao dobro da área do triângulo.

\subsection{Integração Numérica}

Nas equações (9) e (10) os núcleos $u^{*}$ e $q^{*}$ são funções elaboradas, porém suaves, para os casos em que $P$ e $Q$ (ponto de fonte ou de colocação e ponto campo ou integração, respectivamente) não coincidem ou não estão demasiadamente próximos. Caso o ponto de colocação não pertença ao elemento no qual a integral está sendo avaliada, nenhuma singularidade ocorre, conforme a situação mostrada na figura 3 (a) a seguir. Por outro lado, se $P$ pertence ao elemento que está sendo integrado, é possível que $P$ e $Q$ coincidam, o que significa singularidade no integrando, vide figura 3 (b). Uma observação importante consiste no fato de que o ponto pode pertencer a outro elemento, mas ser um nó duplo. Nesta situação também pode ocorrer singularidade, caso o ponto fonte realize a integração sobre o elemento que contenha o seu nó duplo.
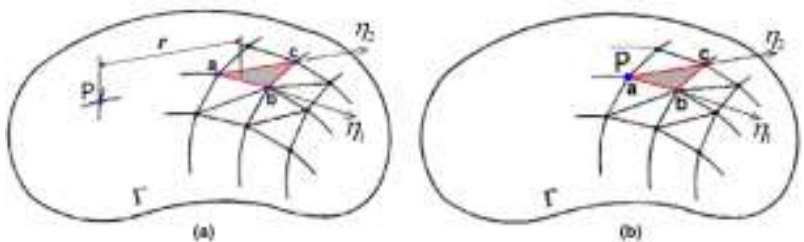

Figura 3 - (a) Elemento regular; (b) Elemento singular.

Faz-se o cálculo numérico das integrais usando a Quadratura de Gauss quando o ponto fonte não se encontra no mesmo elemento em que se processa a integração. Reserva-se a integração analítica apenas para o cálculo dos coeficientes $G_{j}$. Isto porque os coeficientes $H_{j}$, cuja integral é mais fortemente singular, podem ser calculados pela soma dos coeficientes das linhas correspondentes, segundo o procedimento da obediência à imposição de um campo potencial constante [5]. Quando um campo uniforme de potencial é aplicado num domínio físico finito as derivadas do potencial devem ser totalmente nulas:

$[H]\{U\}=\{0\}$

Dessa forma, tanto a integral mais fortemente singular $H_{j}$ quanto o valor do coeficiente $c(P)$ podem ser calculados indiretamente. No MEC, para problemas tridimensionais, a fórmula apropriada da Quadratura de Gauss para triângulos [10] requer avaliar a integral de um domínio triangular, onde $\Delta$ é um triangulo arbitrário:

$I_{1}=\iint_{\Delta} f(x, y) d x d y$

Fazendo a transformação de coordenadas, tem-se:

$I_{2}=|J| \int_{\eta_{1}=0}^{1} \int_{\eta_{2}=0}^{1-\eta_{1}} f(x(\eta), y(\eta), z(\eta)) d \eta_{2} d \eta_{1}$

$\mathrm{Na}$ concepção da solução numérica para as integrais deve-se estabelecer pontos distintamente posicionados 
sobre o elemento triangular, em que se possa determinar o valor da função [7]. Assim, a solução de cada uma das integrais apresenta-se como:

$\iint_{s_{j}} f(x) d S=\sum_{p=1}^{N G} W_{p} f\left(\xi_{p}\right)|J|$

Onde $N G$ é o número de pontos de integração, $W_{p}$ são os pesos da quadratura de Gauss e $\xi_{p}$ são pontos de integração de Gauss, $J$ é o Jacobiano para a transformação de coordenadas e $f\left(\xi_{p}\right)$ é o valor da função a ser integrada, no ponto $P$.

Os elementos das matrizes $G$ e $H$ podem ser escritos das seguinte forma, utilizando as equações (9) e (10).

$$
\begin{aligned}
& {[\hat{H}]=\int_{S_{j}} q^{*} u d S=\left[\sum_{l=1}^{L}\left[q^{*} \phi\right], w_{l}|J|\right] u^{n}} \\
& {[G]=\int_{S_{j}} u^{*} q d S=\left[\sum_{l=1}^{L}\left[u^{*} \phi{ }_{l}\right]_{l}|J|\right] q^{n}}
\end{aligned}
$$

A solução fundamental tridimensional é dada por:

$$
u^{*}=\frac{1}{4 \pi r}
$$

Na equação (19), $r$ é a distância do ponto de aplicação $\xi$ do potencial unitário a um ponto qualquer onde desejamos determinar o valor da função $u^{*}$. A solução fundamental do fluxo é dada por:

$$
q^{*}=\frac{\partial u^{*}}{\partial n}=\frac{\partial u^{*}}{\partial r} \frac{\partial r}{\partial n}=\frac{-\cos (r, n)}{4 \pi r^{2}}
$$

Em que:

$\cos (r, n)=\frac{\mu \cdot h}{r n}$

O cosseno diretor $\cos (r, n)$ dos vetores $r$ e $n$ é representado na figura 4, assim como o vetor $r$, que é a diferença entre as coordenadas dos pontos $i$ e $t$.

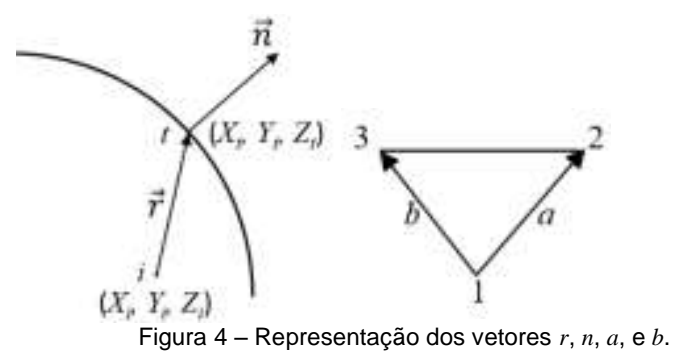

Os parâmetros $r_{1}, r_{2}, r_{3}, n_{1}, n_{2}$ e $n_{3}$ que definem os vetores $r$ e $n$, são facilmente determinados:

$$
r_{1}=x_{t}-x_{i} ; \quad r_{2}=y_{t}-y_{i} ; \quad r_{3}=z_{t}-z_{i}
$$

Para determinar os valores do vetor $n$, supõe-se uma superfície do elemento triangular equipotencial e convenciona-se uma numeração dos seus nós no sentido anti-horário. Desse modo, a normal é ortogonal ao plano definido pelos três nós do elemento e pode ser definida como o produto vetorial entre dois vetores que pertençam ao plano do elemento. Considerando-se que os dois vetores sejam representados por quaisquer dois lados do elemento triangular, indicado pelos lados $a$ e $b$ (vide figura 4) tem-se:

$\rho=\rho \times b$

Para que a normal seja dirigida para fora do domínio, adota-se uma convenção para a numeração dos nós do elemento triangular no sentido anti-horário, conforme figura 4. Desta forma se assegura que para todos os elementos o sentido da normal $n$ será o mesmo, isto é, para fora do domínio. Desta forma escreve-se o vetor $n$ :

$$
\begin{aligned}
& n_{1}=\left(y_{2}-y_{1}\right)\left(z_{3}-z_{1}\right)-\left(z_{2}-z_{1}\right)\left(y_{3}-y_{1}\right) \\
& n_{2}=\left(z_{2}-z_{1}\right)\left(x_{3}-x_{1}\right)-\left(x_{2}-x_{1}\right)\left(z_{3}-z_{1}\right) \\
& n_{3}=\left(x_{2}-x_{1}\right)\left(y_{3}-y_{1}\right)-\left(y_{2}-y_{1}\right)\left(x_{3}-x_{1}\right)
\end{aligned}
$$

Agora pode-se reescrever as equações (17) e (18):

$$
\begin{aligned}
& {[\hat{H}]=\left[\sum_{l=1}^{L}\left[\frac{-\left(r_{1} n_{1}+r_{2} n_{2}+r_{3} n_{3}\right)}{4 \pi r^{3} n} \phi\right]_{l} w_{l}|J| u^{n}\right.} \\
& {[G]=\left[\sum_{l=1}^{L}\left[\frac{1}{4 \pi r} \phi\right]_{l}\left|w_{l}\right| J \mid q^{n}\right.}
\end{aligned}
$$

\subsection{Integração Analítica}

O processo anterior de integração numérica se faz necessário na maioria dos casos, visto que a solução exata é de difícil concepção. Entretanto esta pode ser facilmente determinada nos casos particulares em que:

a) os vetores $r$ e $n$ são ortogonais;

b) o ponto fonte se encontra aplicado em um dos nós do elemento sobre o qual se efetua a integração.

No primeiro caso, o integrando é nulo, pois $r$ e $n$ são ortogonais, ou seja, o cosseno diretor das suas retas suporte é nulo.

No segundo caso, na suposição de que o ponto fonte está aplicado no ponto 1 do elemento triangular, e se estabeleça um sistema local de eixos cartesianos com origem neste ponto e com o eixo y paralelo ao lado 2-3, pode-se, baseado no que indica a figura 5 , escrever as seguintes relações para $\eta_{1}, \eta_{2}$ e $\eta_{3}$. [11], onde $H$ é a altura do elemento triangular relativa ao lado 2-3:

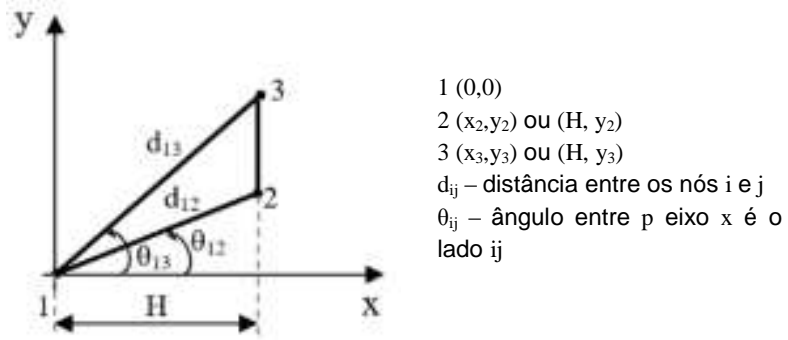

Figura 5 - Pontos do elemento triangular. 
$\eta_{1}=\frac{H-x}{H} ; \quad \eta_{2}=\frac{\frac{y_{3}}{x_{3}} x-y}{y_{3}-y_{2}} ; \quad \eta_{3}=\frac{\frac{y_{2}}{x_{2}} x-y}{y_{2}-y_{3}}$

Expressando tais funções $\eta_{k}$ em coordenadas polares:

$x=r \cos (\theta) ; \quad y=r \operatorname{sen}(\theta)$

Considerando que:

$\frac{y_{2}}{x_{2}}=\operatorname{tg}\left(\theta_{12}\right) ; \quad \frac{y_{3}}{x_{3}}=\operatorname{tg}\left(\theta_{13}\right) ; \quad y_{3}-y_{2}=d_{23}$

As funções $f_{k}=\eta_{k} u^{*}$ são dadas por:

$f_{1}=\frac{H-r \cos \theta}{4 \pi r H}$

$f_{2}=\frac{\operatorname{tg}\left(\theta_{13}\right) \cos \theta-\operatorname{sen} \theta}{4 \pi d_{23}}$

$f_{3}=\frac{-\left(\operatorname{tg}\left(\theta_{12}\right) \cos \theta-\operatorname{sen} \theta\right)}{4 \pi d_{23}}$

As integrais $I_{k}=\int_{S_{j}} f_{k} d x d y$ se apresentam como:

$I_{k}=\iint_{\theta} f_{k} r d r d \theta$

Visto que:

$d x d y=\left|\begin{array}{ll}\frac{\partial x}{\partial r} & \frac{\partial x}{\partial \theta} \\ \frac{\partial y}{\partial r} & \frac{\partial y}{\partial \theta}\end{array}\right| d r d \theta=r d r d \theta$

Assim, tem-se a $1^{\text {a }}$ integral:

$I_{1}=\frac{1}{4 \pi H} \int_{\theta} \int_{r}(H-r \cos \theta) d r d \theta$

Por simples observação na figura 5 , verifica-se que $r$ pode ser representado em função de $\theta$.

$r=\frac{H}{\cos \theta}$

Substituindo a equação (36) na (35), chega-se a:

$I_{1}=\frac{H}{8 \pi} \ln \left|\frac{\sec \theta_{13}+\operatorname{tg} \theta_{13}}{\sec \theta_{12}+\operatorname{tg} \theta_{12}}\right|$

Fazendo o mesmo para as equações (31) e (32):

$$
\begin{aligned}
& I_{2}=\frac{H^{2}}{8 \pi d_{23}}\left(\operatorname{tg} \theta_{13} \ln \left|\frac{\sec \theta_{13}+\operatorname{tg} \theta_{13}}{\sec \theta_{12}+\operatorname{tg} \theta_{12}}\right|+\left(\sec \theta_{12}-\sec \theta_{13}\right)\right) \\
& I_{3}=\frac{-H^{2}}{8 \pi d_{23}}\left(\operatorname{tg} \theta_{12} \ln \left|\frac{\sec \theta_{13}+\operatorname{tg} \theta_{13}}{\sec \theta_{12}+\operatorname{tg} \theta_{12}}\right|+\left(\sec \theta_{12}-\sec \theta_{13}\right)\right)
\end{aligned}
$$

Com base mais uma vez na figura 5 pode-se concluir:

$\cos \theta_{i j}=\frac{H}{d_{i j}} ; \quad \operatorname{tg} \theta_{i j}=\frac{\sqrt{d_{i j}^{2}-H^{2}}}{H} ; \quad \sec \theta_{i j}=\frac{d_{i j}}{H}$

Desta forma indicam-se as expressões de $I_{1}, I_{2}$ e $I_{3}$ :

$$
\begin{aligned}
& I_{1}=\frac{H}{8 \pi} \ln \left|\frac{d_{13}+\sqrt{d_{13}^{2}-H^{2}}}{d_{12}+\sqrt{d_{12}^{2}-H^{2}}}\right| \\
& I_{2}=\frac{H}{8 \pi d_{23}}\left(\sqrt{d_{13}^{2}-H^{2}} \ln \left|\frac{d_{13}+\sqrt{d_{13}^{2}-H^{2}}}{d_{12}+\sqrt{d_{12}^{2}-H^{2}}}\right|+\left(d_{12}-d_{13}\right)\right) \\
& I_{3}=\frac{-H}{8 \pi d_{23}}\left(\sqrt{d_{12}^{2}-H^{2}} \ln \left|\frac{d_{13}+\sqrt{d_{13}^{2}-H^{2}}}{d_{12}+\sqrt{d_{12}^{2}-H^{2}}}\right|+\left(d_{12}-d_{13}\right)\right)
\end{aligned}
$$

Desta forma determinados os termos de $[G]_{i j}$ para o caso em que o potencial unitário está aplicado em um dos nós do elemento triangular sobre o qual se processa a integração.

\section{Exemplo de Aplicação}

O exemplo proposto neste trabalho consiste em um cubo homogêneo engastado na extremidade da face $y$ $z$ onde $x=0$ e sujeito a uma deformação constante aplicada na direção $x$ na face $y-z$ onde $x=1$, conforme mostrado na figura 6.Com a aplicação de condições naturais nulas nas faces dos planos $x-z$ e $x-y$, o problema fica unidimensional. Para discretização foram empregadas sete malhas diferentes com elementos triangulares conforme mostrado na tabela 1:

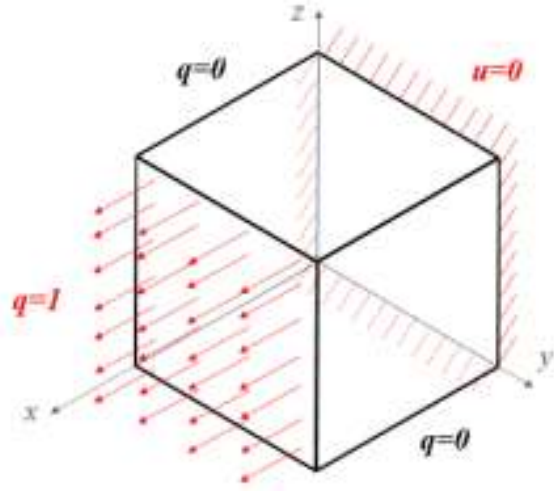

Figura 6 - Geometria e condições de contorno.

Tabela 1: Quantidade de pontos nodais e elementos em cada malha.

\begin{tabular}{|l|c|c|c|c|c|c|c|}
\hline & Malha 1 & Malha 2 & Malha 3 & Malha 4 & Malha 5 & Malha 6 & Malha 7 \\
\hline Pontos & 24 & 54 & 96 & 150 & 294 & 486 & 600 \\
\hline Elementos & 12 & 48 & 108 & 192 & 432 & 768 & 972 \\
\hline
\end{tabular}

$\mathrm{Na}$ figura 7 observa-se o erro médio relativo para o cálculo do potencial onde o fluxo é prescrito, o erro foi muito pequeno desde a malha com o menor refinamento, onde se possui apenas 12 elementos, ou seja dois elementos triangulares por face do cubo.

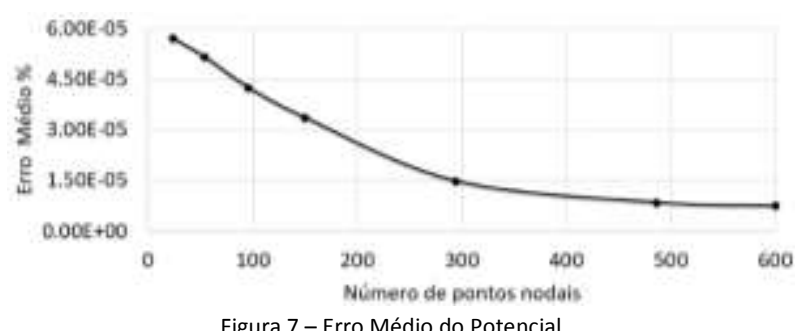


Já na figura 8 o erro médio relativo para o cálculo da derivada potencial é uma ordem de grandeza maior que o erro médio do potencial, mas continua sendo muito pequeno para todas as malhas.

Tanto os erros médios do potencial e da derivada potencial observamos a curva de convergência a medida que a malha foi sendo refinada.

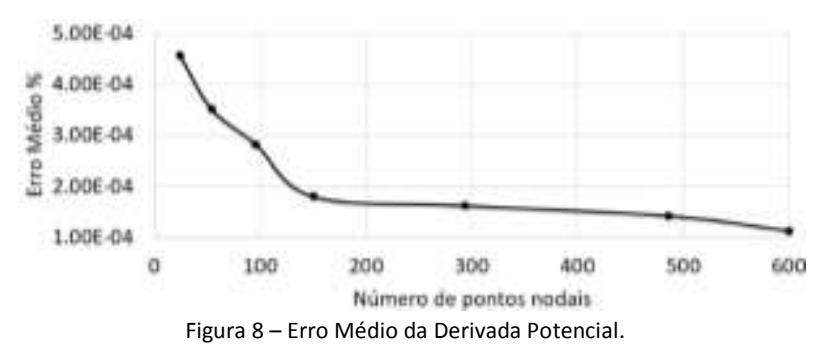

\section{Conclusões}

Diferentemente dos problemas bidimensionais, a descrição de um esquema de discretização e o modus operandi de solução não são evidentes nem unívocos. A literatura especializada não os descreve em pormenor, ficando a cargo do programador a arquitetura de um esquema ao mesmo tempo funcional e consistente de solução.

Os resultados numéricos obtidos mostraram um desempenho satisfatório da metodologia de integração aqui desenvolvida, indicando que o esquema numérico para os elementos sem singularidades e a solução pelo método analítico para as integrações com singularidades pode ser usado com êxito em problemas tridimensionais, mesmo utilizando uma quantidade reduzida de pontos nodais.

Também credencia o modelo descrito a ser utilizado em simulações futuras com conformações geométricas mais intrincadas. Também endossa sua extensão aos problemas pertinentes às Equações de Helmholtz e da Onda Acústica, ou seja, casos em que se deseja determinar a resposta dinâmica ao longo do tempo, pois em ambos os casos o operador Laplaciano figura no modelo matemático.

\section{Referências}

[1] BREBBIA, C. A. The Boundary Element Method for Engineers. London: Pentech Press: 1978.

[2] REDDY, J. An Introduction to the Finite Element Method, 3rd edition. New York: McGraw-Hill: 2005.

[3] LEVEQUE, R. Finite Difference Methods for Ordinary and Partial Differential Equations. Philadelphia: SIAM: 2007.

[4] BREBBiA, C. A., TELLES, J. C. F., WROBEL, L.C., Boundary Element Techniques: Theory and Applications in Engineering. Berlin: Springer - Verlag: 1984.

[5] BREBBIA, C. A.; DOMíNGUEZ J. The Boundary Element Method - An Introductory Course. UK: WIT Press: 1998.

[6] LUIZ, T. F. Aplicação do MEC a problemas de potencial tridimensional em meios heterogêneos. Tese (Doutorado) COPPE/UFRJ, Rio de Janeiro, 2006.

[7] HAMMER, P. C., MARLOWE, O. J., STROUD, A. H. Numerical Integration over Simplexes and Cones, Math. Tables and other Aids to Comp, v. 10, p. 130-137, 1956.

[8] CUROTTO, C. L. Método dos elementos de contorno para elasticidade tridimensional. Dissertação (Mestrado) COPPE/UFRJ, Rio de Janeiro, 1981.

[9] SOUZA, L. H. G., APARECIDO, J. B. Numerical integration by Gauss-Legendre quadrature over triangular domains. The International Congresso of Mechanical Engineering - COBEM, 2007.

[10] HUSSAIN, F., KARIM, M. S., AHAMAD, R. Appropriate Gaussian quadrature formulae for triangles. International Journal of Applied Mathematics and Computation, v. 4(1), p. 24-38, 2012.

[11] SÁ, P. A. C. de. Aplicação do Método dos elementos de contorno a problemas de campo. Dissertação (Mestrado) COPPE/UFRJ, Rio de Janeiro, 1980. 